Research, Society and Development, v. 10, n. 16, e176101623806, 2021

(CC BY 4.0) | ISSN 2525-3409 | DOI: http://dx.doi.org/10.33448/rsd-v10i16.23806

\title{
Avaliação dos aspectos nutricionais e o risco do desenvolvimento de doenças renais crônicas
}

\author{
Aspects and the risk of developing chronic kidney diseases
}

Evaluación de los aspectos nutricionales y el riesgo de desarrollar enfermedades renales crónicas

Recebido: 23/11/2021 | Revisado: 28/11/2021 | Aceito: 29/11/2021 | Publicado: 10/12/2021

\author{
Moisés Iasley Lima Vasconcelos \\ ORCID: https://orcid.org/0000-0002-8724-1063 \\ Centro Universitário Estácio do Ceará, Brasil \\ E-mail: moisesasley@gmail.com \\ Francisca Karoline Da Costa Silva \\ ORCID: https://orcid.org/0000-0001-5477-4130 \\ Centro Universitário Estácio do Ceará, Brasil \\ E-mail: karolinesilva684@gmail.com \\ Helder Matheus Alves Fernandes \\ ORCID: https://orcid.org/0000-0003-2068-9071 \\ Faculdade Nova Esperança de Mossoró, Brasil \\ E-mail:heldermatheus10@hotmail.com \\ Igor de Codes Soares \\ ORCID: https://orcid.org/0000-0002-8311-629X \\ Universidade de Fortaleza, Brasil \\ E-mail: igordecodes97@gmail.com \\ Natan Ivens Castro de Sousa \\ ORCID: https://orcid.org/0000-0001-9863-4594 \\ Universidade Estadual do Ceará, Brasil \\ E-mail: Natan.ivens@aluno.uece.br \\ Fernanda Fernandes de Oliveira Silva \\ ORCID: https://orcid.org/0000-0003-4003-5120 \\ Universidade de Fortaleza, Brasil \\ E-mail: fernanda.fernandes3006@gmail.com \\ Joana Limaverde Araújo \\ ORCID: https://orcid.org/0000-0002-3231-4966 \\ Universidade de Fortaleza, Brasil \\ E-mail: joana_limaverde@ @otmail.com \\ Natália Pereira do Nascimento \\ ORCID: https://orcid.org/0000-0002-6727-3236 \\ Centro Universitário Estácio do Ceará, Brasil \\ E-mail: nataliapn.nutri@gmail.com \\ Carolina Drummond Barboza \\ ORCID: https://orcid.org/0000-0002-7392-2562 \\ Universidade Estadual do Ceará, Brasil \\ E-mail: carolina.cdb@isgh.org.br
}

\begin{abstract}
Resumo
O trabalho teve como objetivo utilizar a literatura como referência para abordar assuntos recentes e pertinentes ao desfecho da doença renal crônica (DRC), com enfoque nutricional. Trata-se de um referencial teórico, cuja busca foi realizada nas bases de dados: PubMed, LILACS, SciELO e REDIB, utilizando os seguintes descritores disponíveis nos Descritores em Ciências da Saúde (DeCS): Doença renal crônica, Nutrição, Terapia nutricional, Paciente renal e Hábitos alimentares. Foram utilizados os operadores booleanos "AND" e "OR", realizando as distintas combinações. A DRC acomete os rins e leva a perda gradual da estrutura e da função renal, consequentemente há aumento da mortalidade e morbidade. A população de risco para DRC são: portadores de Hipertensão arterial, Diabetes Mellitus, doenças cardiovasculares, assim como idosos, fumantes, pessoas com IMC acima de $30 \mathrm{~kg} / \mathrm{m}^{2}$ e que possuam histórico familiar de DRC. No Brasil, mais de dez milhões de pessoas são portadores de DRC. Nesse contexto, é necessário avaliar o impacto alimentar sobre essa doença; alguns nutrientes como proteína, fósforo, potássio e sódio necessitam de recomendações dietéticas especiais. O número de pessoas acometidas por DRC vem crescendo, o que pode levar a distúrbios nutricionais. A correria da vida moderna leva a população a crer que produtos processados e ultraprocessados, são uma boa alternativa devido a sua praticidade, podemos concluir então que trata-se de uma dieta
\end{abstract}


pobre em fibras alimentares e rica em sódio e temperos artificiais. É necessário que se faça uma conscientização da população quanto a hábitos saudáveis, para um melhor desfecho das DRC.

Palavras-chave: Doença renal crônica; Estado nutricional; Risco.

\begin{abstract}
The study aimed to use the literature as a reference to address issues relevant and pertinent to the outcome of chronic kidney disease (CKD), with a nutritional focus. This is a theoretical framework, which was searched in the following databases: PubMed, LILACS, SciELO and REDIB, using the following descriptors available in the Health Sciences Descriptors (DeCS): Chronic kidney disease, Nutrition, Nutritional therapy, Patient kidney and eating habits. The Boolean operators "AND" and "OR" were used, performing as distinct models. CKD affects the kidneys and leads to a gradual loss of renal structure and function, consequently increasing mortality and morbidity. The population at risk for RDC are: patients with hypertension, diabetes mellitus, cardiovascular diseases, as well as elderly, years, people with a BMI above $30 \mathrm{~kg} / \mathrm{m}^{2}$ and who have a family history of RDC. In Brazil, more than ten million people are carriers of the DRC. In this context, it is necessary to assess the food impact on this disease; some nutrients like protein, phosphorus, potassium and content content of special dietary recommendations. The number of people affected by CKD is growing, which can lead to nutritional disorders. The rush of modern life leads people to believe that processed and ultra-processed products are a good alternative due to their practicality, we can conclude that this is a diet low in dietary fiber and rich in sodium and artificial seasoning. It is necessary to make the population aware of healthy habits, for a better outcome of the DRC.
\end{abstract}

Keywords: Chronic kidney disease; Nutritional status; Risk.

\title{
Resumen
}

El estudio tuvo como objetivo utilizar la literatura como referencia para abordar temas relevantes y pertinentes para el desenlace de la enfermedad renal crónica (ERC), con un enfoque nutricional. Se trata de un marco teórico, que se buscó en las siguientes bases de datos: PubMed, LILACS, SciELO y REDIB, utilizando los siguientes descriptores disponibles en los Descriptores de Ciencias de la Salud (DeCS): Enfermedad renal crónica, Nutrición, Terapia nutricional, Riñón del paciente y hábitos alimenticios. . Se utilizaron los operadores booleanos "Y" y "O", que funcionan como modelos distintos. La ERC afecta a los riñones y conduce a una pérdida gradual de la estructura y función renal, lo que aumenta la mortalidad y la morbilidad. La población en riesgo de CDR son: pacientes con hipertensión, diabetes mellitus, enfermedades cardiovasculares, así como ancianos, años, personas con IMC superior a $30 \mathrm{~kg} / \mathrm{m}^{2}$ y que tengan antecedentes familiares de CDR. En Brasil, más de diez millones de personas son portadores de la República Democrática del Congo. En este contexto, es necesario evaluar el impacto de los alimentos en esta enfermedad; algunos nutrientes como proteínas, fósforo, potasio y contenido contenido de recomendaciones dietéticas especiales. El número de personas afectadas por la ERC está aumentando, lo que puede provocar trastornos nutricionales. El ajetreo de la vida moderna lleva a las personas a creer que los productos procesados y ultraprocesados son una buena alternativa debido a su practicidad, podemos concluir que esta es una dieta baja en fibra dietética y rica en sodio y condimentos artificiales. Es necesario concienciar a la población sobre hábitos saludables, para un mejor resultado de la RDC.

Palabras clave: Enfermedad renal crónica; Estados nutricionales; Riesgo.

\section{Introdução}

A doença Renal Crônica (DRC) é definida como uma lesão localizada nos rins, que resulta em perda progressiva e irreversível de todas as funções renais: glomerular, endócrina e tubular, desassociado aos fatores etiológicos presentes que se envolvem nessa patologia, sendo de alta prevalência nos pacientes hospitalizados (Fiaccadori et al., 2021).

Os rins têm várias funções, dentre elas: excreção, filtração, realização de processos metabólicos, síntese hormonal, regulação do equilíbrio hidroeletrolítico e da pressão arterial. O papel excretor dos rins, possui relação com desfechos clínicos, pois todas as funções renais normalmente declinam juntamente a sua função de excretar substâncias (Brasil, 2014).

Independente da etiologia da doença de base, o principal desfecho nos pacientes com DRC são suas complicações: anemia, acidose metabólica, desnutrição e alteração do metabolismo de cálcio e fósforo proveniente da perda da função renal, o óbito e a necessidade de terapia renal substitutiva (Magalhães et al., 2018).

A DRC pode ter consequências sobre o metabolismo, absorção, biodisponibilidade e excreção de nutrientes, dando prevalência para distúrbios nutricionais, o que leva em um desequilíbrio renal e metabólico que atingirá de maneira direta ou indireta o estado nutricional de pacientes com DRC (Cozzolino \& Cominetti, 2019). 
Há uma alta prevalência de DRC no Brasil. Estima-se que aproximadamente 11 a 22 milhões de adultos têm algum grau de disfunção renal, numa população de aproximadamente 200 milhões de habitantes. Essa taxa não pode ser tratada por especialistas, o que mostra a necessidade de programas epidemiológicos específicos e informação ao médico generalista sobre os tratamentos que previnem a progressão da DRC (Sarmento et al., 2018).

A avaliação nutricional possui grande importância nos cuidados de pacientes hospitalizados, pois fornece o diagnóstico do estado nutricional do paciente que influencia de maneira direta no seu desfecho clínico e, nesse viés, o nutricionista deve estar inserido em uma equipe multidisciplinar e interdisciplinar para manusear os protocolos e programas de segurança ao paciente, possibilita a criação de um plano de cuidado integral (Vasconcelos et al., 2021).

A desnutrição afeta negativamente o desfecho da DRC, pois está ligada a uma maior quantidade de complicações, além da maior frequência e duração de internações hospitalares. A origem da desnutrição relacionada a DRC é multifatorial, sendo que a maioria está associada à ingestão alimentar insuficiente e/ou, principalmente, ao hipercatabolismo (Barboza \& Salomon, 2020; Vasconcelos et al., 2021).

A vida moderna trouxe falta de tempo e procura pela praticidade, aliada a isso está o convencimento da população por parte da indústria de que produtos prontos para consumo ou de preparação rápida e fácil, de baixo custo, podem ser uma boa escolha, além de que esses alimentos se tornam mais aceitáveis ao paladar as custas de adição de grandes quantidades de óleo, açúcar e sal, o que leva a um ganho de peso, e consequentemente, desenvolvimento de DRC (Souza, 2019).

Pacientes hospitalizados com DRC tem predisposição a desnutrição. Com a aplicação de metodologias como o Questionários De Frequência Alimentar (QFA), tornou-se viável identificar a ingestão alimentar do paciente de maneira mais específica, isso possibilitou a verificação de alterações nas quantidades e qualidades dos alimentos consumidos (Martins et al., 2011).

A terapia nutricional no paciente com DRC desde que planejada, individualizada e de boa aceitação, possibilitará a manutenção e a melhora do estado nutricional, dessa forma, a avaliação nutricional de pacientes hospitalizados possibilita o diagnóstico do estado nutricional e é positivo para a recuperação e/ou manutenção destes, o que favorece uma intervenção nutricional durante sua internação (Pereira et al., 2020).

O rastreamento da DRC é realizado por muitos fatores interligados, que irão alertar para uma predisposição da doença e, segundo o Instituto Nacional de Saúde Norte Americano, os biomarcadores são definidos como indicadores quantidades de processos biológicos ou patológicos usados para fins de diagnóstico ou de monitorização da terapêutica (Cecconello et al., 2019).

Os métodos aplicados para identificação e diagnóstico de DRC em pacientes são a Taxa de Filtração Glomerular (TFG), o exame de sumário de urina (EAS) e um exame de imagem, preferencialmente a ultrassonografia dos rins e vias urinárias, pois são importantes para o diagnóstico. Recomenda-se o cálculo da TFG para pacientes que apresentam riscos de desenvolver DRC, além de que deve-se dosar sua creatinina sérica e avaliar os valores obtidos (Brasil, 2014).

O presente estudo tem como objetivo utilizar a literatura como referência para abordar assuntos recente pertinente a Doença Renal Crônica.

\section{Metodologia}

O presente estudo trata-se de uma revisão integrativa que busca organizar e agrupar os resultados acerca da Doença Renal Crônica, de maneira abrangente, ordenada e atualizada. O levantamento bibliográfico dessa temática ocorreu entre os meses de agosto de 2021 a outubro de 2021. 
A busca pelos artigos selecionados se deu por meio das bases de dados científicos: PubMed, LILACS, SciELO e REDIB, foram utilizados os seguintes descritores disponíveis nos Descritores em Ciências da Saúde (DeCS): Doença renal crônica, Nutrição, Terapia nutricional e Hábitos alimentares. Foram utilizados os operadores booleanos “AND” e “OR”, realizando diferentes combinações nas bases de dados citadas anteriormente.

Essas bases de dados foram selecionadas por serem as mais abrangentes, relevantes e por conterem um maior alcance de resultados na área da saúde e mais especificamente, na área da nutrição. Foram considerados como critério de inclusão os estudos mais recentes no que diz respeito a Doença Renal Crônica, nos idiomas inglês e português, e que fossem do tipo transversal, descritivo, qualitativo e quantitativo. Os critérios de exclusão foram: relatos de casos, relatos de experiências, estudos randomizados e de abordagem ecológica.

Em seguida foi realizada a leitura dos títulos e resumos para que fossem selecionados aqueles artigos mais relevantes e que mais contribuíssem para a elaboração desse referencial teórico, em seguida foi realizada a leitura integral dos materiais selecionados previamente, para que assim fosse elaborado o presente estudo.

Após o emprego dos critérios de exclusão, o número final de estudos selecionados foi de 33 artigos, dentre eles artigos e livros publicados nos últimos 10 anos, além do Guia alimentar para a população brasileira e outros materiais disponíveis no portal do Ministério da Saúde.

\section{Resultados e Discussão}

\section{Doença Renal Crônica}

A Doença Renal Crônica é explicada através da perda gradual da estrutura e função renal, resultando no declínio progressivo das funções fisiológicas dos rins. A diminuição da função renal associa-se ao aumento da mortalidade, morbidade, limitações na vida diária, incapacidades físicas e o impacto negativo na qualidade de vida (Abreu et al., 2019).

Os rins têm como funções: formação da urina, expulsão dos produtos residuais, ajuste dos eletrólitos, regulagem da estabilidade ácido-básico, administração da pressão arterial, controle da produção de eritrócitos, síntese da vitamina D, eliminação das prostaglandinas, coordena o equilíbrio de fósforo e cálcio dentre outras funções importante para sua funcionalidade (Fiaccadori et al., 2021).

$\mathrm{Na}$ fase inicial das nefropatias, grande parcela dos indivíduos apresenta-se assintomáticos dificultando assim o diagnóstico precoce, em meio à população geral, os grupos alvos mais suscetíveis à manifestarem tais patologias são: portadores de Hipertensão Arterial, Diabetes Mellitus, doenças cardiovasculares, assim como idosos, fumantes, pessoas com índice de massa corporal acima de 30kg/m² (Obesidade Grau I, Grau II e Grau III) e que possuam histórico familiar de DRC (Queiroz et al., 2018).

As DRC's podem ser detectadas inicialmente através de exames laboratoriais simples. O tratamento das doenças de base pode impedir ou retardar a evolução para estágios mais avançados das patologias renais, reduzindo a morbimortalidade e custos posteriores na atenção de alta complexidade. Esta avaliação inicial, no Brasil, conforme preconizado pelo Sistema Único de Saúde (SUS) está inserida na Estratégia de Saúde da Família (ESF), porta de entrada para a rede assistencial e encaminhamento para as especialidades (Maltal et al., 2019).

A identificação precoce da DRC é reconhecida como uma medida importante para retardar sua progressão, todavia, essa enfermidade tem sido subdiagnosticada, em parte devido à falta de conhecimento da definição e classificação dos estágios da doença, bem como pela não utilização de testes simples para o diagnóstico e avaliação funcional da doença. No Brasil, estimativas da prevalência dessa enfermidade são incertas. O conhecimento da prevalência da doença renal crônica entre os brasileiros subsidiará melhor o planejamento de ações preventivas e assistenciais (Maltal et al., 2019). 


\section{Epidemiologia da Doença Renal Crônica}

Segundo dados da Sociedade Brasileira de Nefrologia, a prevalência da doença renal crônica no mundo é de 7,2\% para indivíduos acima de 30 anos e 28\% a 46\% em indivíduos acima de 64 anos. No Brasil, a estimativa é de que mais de dez milhões de pessoas tenham a doença. Desses, 90 mil estão em diálise (um processo de estímulo artificial da função dos rins, geralmente quando os órgãos têm 10\% de funcionamento), número que cresceu mais de 100\% nos últimos dez anos (Brasil, 2021).

O Censo da Sociedade Brasileira de Nefrologia (SBN) continua apontando um acréscimo no número de pacientes em tratamento dialítico (de 122.825 em 2016 para 126.583 em 2017), com 40.307 novos pacientes em 2017 (Thomé, et. al., 2019). Apesar do número crescente de pacientes renais crônicos nos últimos 10 anos, o Brasil ainda não dispõe de dados estatísticos sobre intervenções e gastos com a doença renal em seus estágios precoces (não dialíticos). Os cálculos públicos geralmente referem-se à doença renal em seu estágio dialítico, e contam de que o Sistema Único de Saúde (SUS) gasta mais de dois bilhões de reais anualmente para tratar cerca de 85.000 pacientes (Alcalde \& Kirsztajn, 2017)

\section{Fatores de Risco para Doença Renal Crônica}

Alguns indivíduos apresentam suscetibilidade aumentada para desenvolver DRC, onde são considerados grupos de risco, portadores de hipertensão arterial sistêmica (HAS), diabetes mellitus tipo 2 (DM2), doenças cardiovasculares, doenças autoimunes, idosos, familiares de pacientes portadores de DRC, uso de medicações nefrotóxicas (Amaral, 2018).

Um dos grandes fatores relacionados à causa de mortalidade devido a DRC é o aumento da incidência de morbidade e mortalidade por doenças cardiovasculares (DCV), principalmente, diabetes mellitus (DM) e hipertensão arterial sistêmica (HAS), que podem estar coexistindo no percurso da DRC, indicando um contínuo desafio de saúde que precisam ser enfrentadas com adoção de reformulação das políticas públicas e medidas preventivas (Vasconcelos et al., 2021).

\section{Influência Alimentar na DRC}

Os hábitos alimentares ocidentais são excessivamente ricos em proteínas, gordura saturada e sódio, e pobres em frutas, vegetais, grãos e fibras. Considerando as recomendações da Organização Mundial da Saúde (OMS) para população geral, a mesma preconiza um consumo ideal em torno de $0,83 \mathrm{~g}$ de proteínas $/ \mathrm{kg} / \mathrm{dia}$, no entanto o consumo é bem superior do que o recomendado, mas, surpreendentemente, quando faz-se um paralelo entre o consumo de proteínas para o público geral e para os portadores de nefropatias, acaba não sendo uma restrição severa, e sim uma adequação (Vasconcelos et al., 2021).

É sabido que o consumo de alimentos processados e ultraprocessados contribuem para a elevada absorção de sódio pelo organismo, alimentos in natura ou minimamente processados são aqueles providos diretamente da natureza e que não sofreram nenhuma ou mínimas alterações; alimentos processados são decorrentes da adição de ingredientes como açúcar e sal aos produtos in natura; e alimentos ultraprocessados são produtos cuja fabricação envolve várias etapas de processamento em escala industrial, com adição de conservantes, estabilizantes, edulcorantes, entre outros (Machado \& Adami, 2019).

Em resposta à vida moderna, a falta de tempo e o apelo à praticidade, a indústria consegue convencer a população com a proposta de produtos prontos para consumo ou de preparação rápida e fácil, de baixo custo, tornando-os aceitáveis ao paladar às custas da adição de grandes quantidades de óleo, açúcar e sal, ocasionando o aumento de peso e o surgimento de DRC (Souza, 2019).

Diante dessa realidade, o Guia Alimentar para População Brasileira recomenda a utilização moderada do sal de cozinha, e o baixo consumo de alimentos processados e ultraprocessados, logo alimentos in natura podem ser considerados estratégias de prevenção, controle e tratamento para a DRC (Brasil, 2014). 
Os métodos de avaliação do consumo alimentar mais comumente utilizados em estudos epidemiológicos são recordatório de $24 \mathrm{~h}$ (R24h), o registro alimentar (RA) e o questionário de frequência alimentar. O R24h é o método mais utilizado para investigação do consumo alimentar. Esse biomarcador é um instrumento quantitativo, baseado em uma entrevista conduzida por profissional treinado (nutricionista) com o objetivo de colher informações sobre a quantificação do consumo alimentar no período de referência (Cuppari, 2019).

\section{Terapia Nutricional na Doença Renal Crônica}

A nutrição desempenha papel importante no tratamento dos pacientes com DRC, no entanto, existem grandes problemas nutricionais afetando esses pacientes, por exemplo, dietas restritivas. A dieta prescrita ao portador de DRC deve conter calorias e proteínas adequadas, ao mesmo tempo em que se limita a determinados nutrientes, como: cálcio, potássio e fósforo. O aconselhamento nutricional dietético auxilia no controle e na prevenção das complicações da DRC (Cuppari, 2019).

$\mathrm{Na}$ DRC, alguns nutrientes como proteína, fósforo, potássio e sódio requerem cuidados especiais. A elevada ingestão de proteínas influencia a hemodinâmica renal, aumenta o fluxo plasmático renal, a pressão intraglomerular e a TFG. Este efeito fisiológico tem como objetivo aumentar a excreção de produtos derivados do metabolismo das proteínas pelos rins. A dieta hipoprotéica também contribui para a redução da produção endógena e do acúmulo de metabólitos tóxicos (Riella \& Martins, 2013).

Também devemos dar atenção ao ajuste da ingestão de fósforo pela dieta, principalmente nos casos de hiperfosfatemia, outro micronutriente que devemos considerar durante a DRC é o potássio, em estágios avançados da patologia onde há prevalência de hipercalemia. A ingestão de sódio é elevada por pacientes com DRC, a dificuldade para ajustar a ingestão de sódio consiste não apenas em reduzir o consumo de alimentos processados, mas também de cloreto de sódio e temperos artificiais usados no preparo das refeições no dia a dia (Krause et al., 2018).

Os tratamentos utilizados em pacientes nos quais a função renal esteja reduzida a ponto de impedir a manutenção do funcionamento normal do organismo são chamados de Terapias Renais Substitutivas (TRS), caso não haja uma intervenção eficaz nesse estágio pode ser fatal ao paciente. As TRS incluem: Terapias dialíticas, Transplante renal, Hemodiálise e Diálise Peritoneal. A terapia dialítica deve ser indicada em pacientes com DRC quando o rim não consegue desempenhar suas funções primárias e/ou sem resposta às medidas de orientação dietética (Krause et al., 2018).

No transplante renal, um rim saudável é doado a um paciente portador de insuficiência renal crônica avançada por uma pessoa viva ou falecida. Esse rim é implantado através de uma cirurgia no paciente receptor e passa a exercer as funções de filtração e eliminação de líquidos e toxinas. A Hemodiálise requer acesso permanente à corrente sanguínea através de fístula cirúrgica conectando assim uma artéria a uma veia. Já a DP, faz o uso do Peritônio, cirurgicamente um cateter é implantado no abdome e no interior da cavidade peritoneal, ocorrendo assim uma difusão no qual leva os produtos de excreção do sangue através da membrana do peritônio e do meio interno do dialisado, após o descarte do líquido retirado, uma nova solução é inserida (Riella \& Martins, 2013).

\section{Rastreamento da Doença Renal Crônica}

No Brasil já é nítido que a maior parte dos médicos utiliza como biomarcador a creatina sérica, que na prática diária está facilmente disponível para a avaliação da função renal de pacientes. Ainda que não obrigatório no Brasil, diferente de outros países, diversos laboratórios atualmente divulgam estimativas da TFG em seus laudos, mesmo que não seja exigido (Magacho, 2014). 
As alterações parenquimatosas necessitam ser pesquisadas por meio do Exame sumário de Urina (EAS) ou através da Pesquisa de albumina na urina. Todos os pacientes com risco de DRC devem ser submetidos ao EAS. Quando o EAS apresenta em de proteinúria em pacientes diabéticos e hipertensos, é indicada a realização de pesquisa de albumina em amostra isolada de urina corrigida pela creatininúria, a relação Albuminúria - Creatininúria, para indivíduos com histórico de DRC na família, infeccção urinária de repetição e doenças urológicas devem ser realizada Avaliação de imagem (Brasil, 2014).

Seja na fase pré-dialítica ou dialítica, a DRC impõe desafios clínicos intrinsecamente ligados ao estado nutricional. Desnutrição e obesidade são fatores ligados tanto com a incidência quanto a progressão da patologia relacionada ao estado nutricional do paciente com DRC. A manutenção de um estado nutricional adequado, assim como o rastreamento prévio da doença, é indispensável para melhorar a qualidade de vida do enfermo retardando possíveis condições extremas do estado nutricional, que se traduzem em prognósticos negativos na fase não dialítica, pois podem acelerar a perda da função renal e promover a entrada do paciente na terapia renal substitutiva (TRS) (Ferreira et al., 2017).

A ocorrência de desnutrição calórico proteica deve ser evitada, pois possui impacto desfavorável no prognóstico da DRC. As maiores taxas de mortalidade e hospitalização em pacientes submetidos a tratamentos dialíticos está associada com a ocorrência de hipoalbuminemia (desnutrição). É causada por fatores que envolvem: alterações no metabolismo das proteínas, diminuição espontânea da ingestão proteica secundária à perda do apetite, produção hepática reduzida, proteinúria maciça e o estado inflamatório urêmico (Silva et al., 2016).

Recentemente, por ocasionar alterações hemodinâmicas, estruturais, histológicas, assim como desordens metabólicas e bioquímicas que vão ao encontro com agressão renal, a obesidade foi associada com fator independente para o desenvolvimento de DRC. A relação entre obesidade e DRC é interligada a fatores como a resistência à insulina, inflamação, estresse oxidativo e hiperlipidemia (Ferreira et al., 2017).

\section{Avaliação Nutricional de Pacientes com Doença Renal Crônica}

A American Dietetic Association determina a avaliação nutricional como uma abordagem para diagnóstico do estado nutricional com a utilização de avaliação física, históricos nutricionais, medicações, exames laboratoriais e antropométricos. Com objetivo de reconhecer o risco nutricional e realizar aplicações de medidas preventivas, inicia-se o rastreamento nutricional através de protocolos de triagem (Barbosa \& Salomon, 2020).

Para prevenir, diagnosticar e tratar a desnutrição em pacientes renais, o acompanhamento do estado nutricional deve fazer parte da rotina hospitalar, sendo um aspecto fundamental. Os métodos utilizados durante uma avaliação nutricional podem ter duas classificações de acordo com sua abordagem, sendo: objetivos ou subjetivos (Ferreira et al., 2017).

A exemplo de exames objetivos, temos os exames antropométricos laboratoriais, exames clínicos nutricionais, Densitometria e Bioimpedância. Por outro lado, temos como exemplos de métodos subjetivos a Avaliação Subjetiva Global, semiologia nutricional e a Avaliação muscular Subjetiva. É necessário a utilização de métodos de coleta e procedimentos diagnósticos para possibilitar a determinação do estado nutricional para realizar a avaliação nutricional de um grupo de indivíduos (Fontes et al., 2018).

Caracterizada por deficiência ou excesso de um ou mais nutrientes essenciais que, por sua vez, alteram a composição corporal com a diminuição de massa muscular, a desnutrição é uma condição clínica que pacientes portadores de DRC estão predispostos a desenvolver. Sendo assim, quando a ingestão é reduzida, fazendo com que a alimentação seja inábil em calorias e nutrientes, a desnutrição é classificada como primária. Pode ser classificada como secundária quando a alimentação se torna insuficiente porque a necessidade energética está aumentada, seja por verminoses, câncer, anorexias, dentre outras. Além disso, 
também pode ser classificada como desnutrição terciária que é ocasionada pelo aumento do tempo de permanência em hospitalização (Ferreira et al., 2017).

O aumento da taxa de ureia no sangue, que ocorre devido à perda da função exócrina, pode causar desnutrição, provocando um estado inflamatório constante que predispõe a esta condição. Além disso, no período intradialítico as retrições alimentares e a perda de aminoácidos podem levar a ocorrência de diminuição dos níveis de albumina, anoxeria, intercorrências infecciosas, distúrbios gastrointestinais, e administração de certos medicamentos, pois favorecem anormalidades no perfil nutricional dos pacientes (Santos, et al., 2018).

O alto índice de massa corporal (IMC) é um forte fator de risco para DRC e pode ser modificado através da alimentação, sendo assim, a importância da nutrição no cuidado com a saúde do paciente renal é evidenciada desde o contexto das medidas preventivas. O tratamento conservador ou pré-diálise é consistido em atenuar o desenvolvimento da doença e prevenir a desnutrição, assim como minimizar a toxicidade urêmica (Santos et al., 2018).

\section{Exames Bioquímicos para o Diagnóstico da Doença Renal Crônica}

Por meio de exames laboratoriais rotineiros, como a dosagem de creatinina sanguínea e a taxa de filtração glomerular, o diagnóstico precoce da DRC pode ser realizado. O teste mais utilizado para a avaliação da função renal é a dosagem de creatinina, sendo associado para estimar a TFG na triagem de DRC. Ela é um produto residual do metabolismo da creatina e fosfocreatina presentes, principalmente na musculatura esquelética. Nesse sentido, pessoas com maior massa muscular tendem a ter maior excreção de creatinina, ocorrendo especialmente na via renal, de forma fisiológica, sendo $85,0 \%$ por filtração glomerular e $15 \%$ por secreção tubular (Abreu et al., 2019).

Definida como a capacidade dos rins de depurar determinadas substâncias a partir do sangue, a TGF é expressa como o volume de plasma que pode ser completamente depurado. De uma maneira geral, enquanto previne a perda de solutos específicos, proteínas e os componentes celulares do sangue, o rim filtra aproximadamente $120 \mathrm{ml} / \mathrm{min}$ de sangue e o depura de produtos metabólitos nitrogenados (Maltal et al., 2019).

Apesar de ser muito utilizada como um excelente biomarcador laboratorial para a DRC e IRA, é de conhecimento dos profissionais da área de saúde geral que, a creatinina é um marcador que se eleva de forma tardia a nível sanguíneo em pacientes acometidos destas condições clínicas como a DRC e IRA (Abreu et al., 2019).

\section{Considerações Finais}

Levando em consideração os aspectos mencionados, é notório o número crescente de pessoas acometidas por DRC o que interfere negativamente no metabolismo, absorção, biodisponibilidade e excreção de nutrientes, promovendo possíveis distúrbios nutricionais. É necessário que haja uma atenção redobrada da população de risco, ou seja, aquela acometida por doenças como Diabetes Mellitus tipo 2 (DM2), Doenças Cardiovasculares, Doenças Autoimunes, população idosa e que possuem familiares portadores de DRC.

Outro ponto importante a ser considerado são os hábitos alimentares do ocidente, visto que a correria da vida moderna e a necessidade da praticidade leva a população a acreditar que produtos prontos pode ser uma boa alternativa, assim trata-se de uma dieta pobre em alimentos in natura, como frutas, grãos e vegetais o que afeta diretamente a ingestão de fibras alimentares e rica em alimentos processados e ultraprocessados, ricos em conservantes, corante e principalmente, sódio e temperos artificiais.

Desta maneira, é imprescindível que haja a prevenção dessa patologia por meio de conscientização da população quanto à dieta e hábitos saudáveis, dado que alimentos in natura podem ser considerados estratégias de prevenção, controle e 
tratamento para a DRC e quando necessário é fundamental que haja a busca de atendimento médico, além do rastreamento e monitoramento de possíveis casos de DRC, para que desta forma seja feito o tratamento mais adequado para cada estágio. É de fundamental importância que sejam realizados mais estudos acerca desse tema, com intuito de contribuir ainda mais para a comunidade científica.

\section{Referências}

Abreu, L. A., Carvalho, G. A. B., de Azeredo, B. A., Mangabeira, R. R., de Souza, D. C., Guimarães, F. P., \& Bastos, M. A. P. (2019). Importância do diagnóstico precoce da Doença Renal Crônica: uma revisão de literatura: uma revisão de literatura. Revista Atenas Higeia, 1(2), 19-23.

Alcalde, P. R., \& Kirsztajn, G. M. (2018). Gastos do Sistema Único de Saúde brasileiro com doença renal crônica. Brazilian Journal of Nephrology, 40, 122129.

Amaral, T. L. M., Amaral, C. D. A., Vasconcellos, M. T. L. D., \& Monteiro, G. T. R. (2019). Prevalência e fatores associados à doença renal crônica em idosos. Revista de Saúde Pública, 53.

Barbosa, C. M., \& Salomon, A. L. R. (2020). A avaliação Nutricional como fator interveniente na redução da prevalência de desnutrição hospitalar.

Brasil. (2019). Dia Mundial do Rim 2019: Saúde dos Rins Para Todos. Ministério da Saúde.

Brasil. (2014a) Ministério da Saúde. Secretaria de Atenção à Saúde. Departamento de Atenção Especializada e Temática. Diretrizes Clínicas para o Cuidado ao paciente com Doença Renal Crônica - DRC no Sistema Único de Saúde/ Ministério da Saúde. Secretaria de atenção à saúde. departamento de atenção especializada e temática. 37.

Brasil. (2014b) Ministério da Saúde. Secretaria de Atenção à Saúde. Departamento de Atenção Básica. Guia alimentar para a população brasileira / Ministério da Saúde, Secretaria de Atenção à Saúde, Departamento de Atenção Básica.

Cecconello, L., Winkelmann, E. R., Morais, E. M., Krug, R. R., \& Moreira, P. R. (2019). Perfil Clínico-Epidemiológico dos Doentes Renais Crônicos em Tratamento Hemodialítico: um estudo da região noroeste do estado do Rio Grande do Sul, 19(3).

Cozzolino, S. M., \& Cominetti, C. (2019). Bases bioquímicas e fisiologica da nutrição: nas diferentes fases da vida, na saúde e na doença. Manole.

Cuppari, L., (2019). Guia de Nutrição: nutrição clínica no adulto. (4a ed.). Manole.

Ferreira, T., Cavalcanti, J. P. R., Guimarães, R. R., Filho, W. F., Martins, I. M. L., Oliveira, M. V. M. (2017). Avaliação de Fatores de Risco Associados à Doença Renal Crônica em uma Unidade de Saúde. Revista Bionorte, 6(2).

Fiaccadori, E., Sabatino, A., Barazzoni, R., Carrero, J. J., Cupisti, A., Waele, E., Jonckheer, J., Singer, P., \& Cuerda, C. (2021) European Society for Clinical Nutrition and Metabolis - ESPEN: guideline on clinical nutrition in hospitalized patients with acute or chronic kidney disease, Clinical Nutrition.

Fontes, B. C. Anjos, J. S. Black, A. P. Moreira, N. X., \& Mafra, D. (2018) Effects of Low-Protein Diet on lipid and anthropometric profiles of patients with chronic kidney disease on conservative management. Braz. J. Nephrol, 40(3), 225-232.

Krause, M. V., Mahan, L. K., \& Escott-Stump, S. (2018). Krause. Alimentos, Nutrição e Dietoterapia. (14a ed.) GEN Guanabara Koogan.

Machado, S. C., \& Adami F. S. (2019). Relação de Consumo de Alimentos In Natura, Processados e Ultraprocessados com Gênero, Idade e Dados Antropométricos. Revista Brasileira de Obesidade, Nutrição e Emagrecimento, 13 (79), 407-416.

Magacho, E. J. C. (2014) Rastreamento de Doença Renal Crônica: validação do questionário SCORED, nomograma para estimativa da taxa de filtração glomerular e avaliação dos marcadores funcional e de lesão do parênquima renal.

Magalhães, F. G. Goulart, R. M. F, \& Prearo, L.C. (2018) Impacto de um programa de intervenção nutricional com idosos portadores de doença renal crônica. Ciência \& Saúde Coletiva [online], 23(8).

Maltal, D. C., Machado, I. E., Perira, C. A., Figueiredo, A. W., Aguiar, L. K., Almeida, W. S., Souza, M. F. M., Rosenfeld, L. G., \& Szwarwald, C. L. (2019) Avaliação da função renal na população adulta brasileira, segundo critérios Laboratoriais da Pesquisa Nacional de Saúde.

Martins, C., Cuppari L., Avesani, C., \& Gusmão, M. H. (2011) Terapia Nutricional para Pacientes na Fase Não-Dialítica da Doença Renal Crônica. Projeto Diretrizes - Associação Médica Brasileira e Conselho Federal de Medicina - Sociedade Brasileira de Nutrição Parenteral e Enteral Associação Brasileira de Nutrologia.

Pereira, R. A., Ramos, C. I., Teixeira, R. R., Muniz, G. A. S., Claudino, G., \& Cuppari, L. (2020). Diet in Chronic Kidney Disease: an integrated approach to nutritional therapy. Revista da Associação Médica Brasileira, 66(1), 59-67.

Queiroz, M. D. S. R., de Andrade, T. V. F., de Sousa Barros, D., da Silva Alves, H., da Silva, R. O. N., Felício, I. M., \& Dantas, D. S. (2018). Avaliação da função renal em pacientes portadores de doenças crônicas não transmissíveis. Journal of Biology \& Pharmacy and Agricultural Management, 13(3).

Riella MC \& Martins MC. (2013) Nutrição e o rim. Editora Guanabara Koogan. 
Research, Society and Development, v. 10, n. 16, e176101623806, 2021 (CC BY 4.0) | ISSN 2525-3409 | DOI: http://dx.doi.org/10.33448/rsd-v10i16.23806

Santos, K. K., Lucas, T. C., Glória, J. C. R., Pereira, A. C. J., Ribeiro, G. C., Oliveira, M. L. (2018) Perfil Epidemiológico de Pacientes Renais Crônicos em Tratamento. Rev enferm UFPE on line.

Sarmento, L. R., Fernandes, P. F. C. B. C., Pontes, M. X., Correia, D. B. S., Chaves, V. C. B., Carvalho, C. F. D. A., \& Moliterno, L. A. A. (2018). Prevalência das causas primárias de doença renal crônica terminal (DRCT) validadas clinicamente em uma capital do Nordeste brasileiro. Brazilian Journal of Nephrology, 40, 130-135.

Silva, A. T., Fuhr, L. M., \& Wazlawik, E. (2016). Associação entre o escore de desnutrição-inflamação e indicadores do estado nutricional em pacientes submetidos à hemodiálise.

Souza, I. S. (2019). Questionário de frequência alimentar de alimentos com alto teor de sódio: uso do método das tríades na sua validação.

Vasconcelos, M. I. L., Fernandes, H. M. A., da Silva Barbosa, E., de Oliveira Grangeiro, R. F., de Sena, D. B. G., Lopes, V. C. B., \& Firmino, L. A. R. G. (2021). Nutrição e doença renal crônica (DRC): Apresentação das novas recomendações e padrões alimentares conforme as últimas evidências científicas. Research, Society and Development, 10(6), e28610615891-e28610615891. 